\title{
La alimentación para pacientes con diabetes mellitus de tipo 2 en tres hospitales públicos de Cundinamarca, Colombia
}

\author{
Ana Yibby Forero, Jenny Alexandra Hernández, Sandra Milena Rodríguez, Jhon Jairo Romero, \\ Gina Emely Morales, Gabriel Ángel Ramírez \\ Grupo de Nutrición, Subdirección de Investigación Científica y Tecnológica, \\ Dirección de Investigación en Salud Pública, Instituto Nacional de Salud, Bogotá, D.C., Colombia
}

\begin{abstract}
Introducción. En el mundo y en Colombia, la diabetes mellitus de tipo 2 es una de las principales causas de morbimortalidad, y afecta principalmente a los hombres y mujeres mayores de 45 años. Un control inapropiado de la enfermedad puede desembocar en mayores complicaciones de salud y convertirla en una de las de más alto costo. El tratamiento incluye otros componentes, además del control de la glucemia, así como un seguimiento constante del personal médico, y actividades educativas dirigidas al paciente y a su familia.

Objetivo. Caracterizar la alimentación de pacientes con diabetes mellitus de tipo 2 en tres hospitales públicos de Cundinamarca.

Materiales y métodos. Se hizo un estudio descriptivo y transversal con 212 pacientes, el cual incluyó el análisis sociodemográfico, el de la frecuencia del consumo de alimentos y las prácticas alimentarias. Resultados. La mayoría de la población era mayor de 45 años y tenía un bajo nivel educativo; el $52 \%$ contaba con un plan de alimentación, y solo el 8,9 \% recibía orientación nutricional a cargo de un nutricionista. Predominó el consumo de carbohidratos y un bajo consumo de proteínas, vitaminas, minerales y fibra. El consumo de azúcares era menor en los pacientes que tenían un plan dietético $(p<0,05)$.

Conclusiones. La mayoría de los pacientes no recibía una alimentación adecuada. Se evidenció un deficiente cumplimiento del tratamiento dietético, así como limitaciones en la asesoría nutricional y barreras de acceso a los alimentos. Estos resultados contribuirán a mejorar las políticas de prevención y control de la diabetes mellitus de tipo 2 y las estrategias que tengan en cuenta el contexto sociocultural de los pacientes.
\end{abstract}

Palabras clave: diabetes mellitus de tipo 2; alimentación; adulto; dieta. doi: https://doi.org/10.7705/biomedica.v38i3.3816

Feeding in adults with type II diabetes mellitus in three public hospitals in Cundinamarca, Colombia

Introduction: Type II diabetes mellitus (DMII) is one of the main causes of morbidity and mortality both worldwide and in Colombia. It mainly affects men and women over 45 years of age. Inadequate control of this disease can generate greater health complications making it one of the most costly diseases in the country. Treatment includes activities other than glycemic control and requires constant monitoring by medical personnel and educational processes directed at the patient and his or her family.

Objective: To characterize the feeding of patients with DMII in three public hospitals of Cundinamarca. Materials and methods: We conducted a cross-sectional descriptive study of 212 patients that analyzed sociodemographic characteristics, frequency of consumption and feeding practices.

Results: Most of the population was over 45 years old and had a low educational level. In total, 52\% had a dietary plan, but only $8.9 \%$ received nutritional guidance from a nutritionist. The consumption of carbohydrates predominated, with low consumption of proteins, vitamins, minerals, and fiber. The consumption of sugars was lower in patients with a dietary plan $(p<0.05)$.

Conclusions: Most of the patients did not have an adequate nutrition; moreover, there was low adherence to dietary treatment, weakness in nutritional counseling and barriers to food access. These results can contribute to improving policies for the prevention and control of DMII and strategies based on the sociocultural context of the patients.

Key words: Diabetes mellitus, type 2; feeding; adult; diet. doi: https://doi.org/10.7705/biomedica.v38i3.3816

Contribución de los autores:

Ana Yibby Forero: coordinación técnica y administrativa del proyecto, elaboración de la propuesta y divulgación de resultados Jenny Alexandra Hernández y Sandra Milena Rodríguez: elaboración de la propuesta de investigación, recolección y análisis de resultados

Jhon Jairo Romero y Gina Emely Morales: análisis estadístico

Gabriel Ramírez: captura y procesamiento de la información 
Las enfermedades crónicas constituyen la principal causa de muerte y discapacidad en el mundo (1). En América, la diabetes mellitus es uno de los mayores problemas de salud pública y su prevalencia aumenta como consecuencia de los cambios culturales, la disminución de la actividad física y la transición a la ingestión de una dieta calórica (2). En Colombia, las enfermedades crónicas son una de las diez primeras causas de mortalidad en adultos mayores de 45 años, con una tasa de mortalidad promedio entre 2009 y 2011 de 10,4 por 100.000 habitantes, y son, además, las responsables de la mayor proporción de pérdida de años de vida saludable en esta población (3). Por su naturaleza crónica, la gravedad de las complicaciones y los medios que se requieren para su control, la diabetes mellitus de tipo 2 es una enfermedad muy costosa (4). El diagnóstico temprano y el buen control de la diabetes reducen la progresión de las complicaciones crónicas de la enfermedad (5).

El cambio en los estilos de vida es fundamental para el manejo de los pacientes con diabetes, en quienes se debe controlar el consumo de alcohol y tabaco, promover la actividad física y una alimentación saludable con restricción del consumo de algunos nutrientes, aspectos esenciales del tratamiento y la prevención de las complicaciones propias de la enfermedad. El cumplimiento del tratamiento de la diabetes es difícil debido a estos cambios en el estilo de vida (6). La intervención alimentaria no solo debe lograr un buen control glucémico, sino también prevenir los factores de riesgo cardiovascular, como la hipertensión arterial sistémica, la dislipidemia y el exceso de peso, con el fin de disminuir las complicaciones a corto y largo plazo (7).

La dieta baja en fibra y con un alto índice glucémico se ha asociado con un mayor riesgo de diabetes (8); asimismo, los ácidos grasos específicos de la dieta pueden afectar diferencialmente la resistencia a la insulina y el riesgo de diabetes $(9,10)$. Por otra parte, una dieta rica en ácidos grasos monoinsaturados previene la modificación

$\overline{\text { Correspondencia: }}$

Ana Yibby Forero, Grupo de Nutrición, Subdirección de Investigación Científica y Tecnológica, Instituto Nacional de Salud, Avenida calle $26 \mathrm{~N}^{\circ}$ 51-20, segundo bloque, segundo piso, Bogotá, D.C., Colombia

Teléfono: (571) 2207700 , extensión 1222

aforero@ins.gov.co y yibbyforero@gmail.com

Recibido: 24/02/17; aceptado: 01/03/18 oxidativa de las lipoproteínas más que aquella rica en ácidos grasos poliinsaturados; el consumo de aceite de oliva, por ejemplo, reduce la oxidación de las lipoproteínas de baja densidad (lowdensity lipoproteins, LDL) mediada por los ácidos grasos n-3 en pacientes con enfermedad vascular periférica (11).

La capacidad y el compromiso de los pacientes, y la consideración debida a sus condiciones, son fundamentales para garantizar el éxito de la intervención. El adecuado manejo nutricional de las personas beneficia la salud de los pacientes, pero, además, contribuye a disminuir los costos hospitalarios derivados de las complicaciones y del tratamiento de la enfermedad (12). En Colombia, no hay mucha información específica sobre las prácticas alimentarias en el manejo de la diabetes mellitus de tipo 2, y las recomendaciones asociadas con la dieta contempladas en la guía de atención de la enfermedad (13) son muy generales y no se tiene en cuenta el contexto del paciente, lo que limita el cumplimiento de las recomendaciones alimentarias.

En este contexto, el objetivo de este estudio fue caracterizar la alimentación y la coherencia con el plan dietético en los pacientes adultos con diabetes mellitus de tipo 2 que asisten al programa de control en tres hospitales públicos de Cundinamarca, Colombia, para recabar información que permita ajustar las estrategias de intervención alimentaria de manera que aumente su efecto positivo en el cumplimiento del tratamiento y contribuya a la disminución de las complicaciones y de la tasa de mortalidad.

\section{Materiales y métodos}

Se hizo un estudio descriptivo y transversal desarrollado en los hospitales San José, del municipio de Guaduas, San Rafael, del municipio de Facatativá y el Universitario La Samaritana, del municipio de Zipaquirá, en Cundinamarca.

En la muestra se incluyeron todos los pacientes atendidos por el programa de control de la diabetes para, así, garantizar la confiabilidad y la precisión de las estimaciones y la validez estadística de los resultados. Se seleccionaron aquellos pacientes con diagnóstico de diabetes mellitus de tipo 2 sin complicaciones derivadas de la enfermedad en los últimos seis meses y se excluyeron las mujeres en periodo de gestación. La muestra final estuvo compuesta por 212 adultos entre los 25 y 70 años que participaron voluntariamente y firmaron el consentimiento informado. 
Se usó un cuestionario general sobre las variables sociodemográficas, las de alimentación, la prescripción del plan dietético y un formulario de frecuencia de consumo validado a partir del modelo utilizado en la Encuesta Nacional de la Situación Nutricional en Colombia (ENSIN, 2010) (14), y adaptado para los fines del presente estudio.

La lista de 28 alimentos se elaboró a partir de los grupos definidos en las guías alimentarias para la población colombiana (15) y se centró en las fuentes de energía, las proteínas, las vitaminas y los minerales, así como en los alimentos considerados poco saludables, como las comidas rápidas, los snacks, y los alimentos con altos contenidos de azúcar y grasas, entre otros, contemplando un período de recordación de 30 días y diez categorías de frecuencia: nunca, una vez al mes, dos a tres veces al mes, una vez por semana, dos veces por semana, tres a cuatro veces por semana, cinco a seis veces por semana, una vez al día, dos veces al día, y tres o más veces al día (14). Además, se indagó por los tiempos de las comidas, las preferencias alimentarias, el consumo de aceites de origen vegetal de diversas fuentes, el consumo de sal, el hábito de retirar la grasa visible de las carnes, el seguimiento del plan dietético y la capacidad de compra de alimentos.

Los principales estimadores estadísticos obtenidos fueron los indicadores de proporción (distribución binomial), para cada uno de los cuales se estimó el error de muestreo y su correspondiente intervalo de confianza de $95 \%$. Con estos, se determinó el nivel de precisión de las estimaciones a partir de la muestra obtenida. Para las comparaciones, se construyeron pruebas de diferencia con la prueba , con un nivel se significación de $a=0.05 \mathrm{si}$ las dos proporciones eran o no estadísticamente iguales. En la estimación de los indicadores y en las pruebas de diferencia, se utilizó el programa Stata $13.1^{\mathrm{TM}}$.

\section{Consideraciones éticas}

El presente estudio se ajustó a las normas científicas, técnicas y administrativas de la investigación en salud establecidas en la resolución $\mathrm{N}^{\circ} 008430$ de 1993 en Colombia, según las cuales se trató de una investigación sin riesgo basada en encuestas que no requerían ninguna intervención o modificación intencionada de las variables biológicas, fisiológicas, sicológicas o sociales de los participantes, quienes, además, firmaron el consentimiento informado.
El proyecto fue aprobado por el Comité de Ética y el Comité Técnico del Instituto Nacional de Salud.

\section{Resultados}

La mayoría de la población estaba entre los 45 y 65 años (58 \%): el $37 \%$ mayor de 65 años y el $6 \%$, menor de 45 años; el $24 \%$ de los pacientes era de sexo masculino y el $76 \%$, de sexo femenino. El periodo de evolución de la enfermedad era de 7,9 años en promedio. Más del $90 \%$ de los pacientes pertenecía a los niveles 1 y 2 del Sisbén y casi todos (99\%) estaban en el régimen subsidiado de salud. El 77,7\% de ellos vivía en el área urbana y el resto en la zona rural. En cuanto a la escolaridad, el $64 \%$ de los participantes había cursado la educación básica primaria sin diferencias por sexo $(p<0,05)$; el $11 \%$ de los hombres y el $19 \%$ de las mujeres no tenía ningún tipo de formación educativa, y de estos, el $77 \%$ no sabía leer ni escribir. El promedio de ingresos era de menos de la mitad de un salario mínimo legal vigente, lo cual constituye una dificultad evidente para acceder a los alimentos: el $44 \%$ ocasionalmente contaba con dinero para comprar verduras y frutas, mientras que el $12 \%$ manifestó no tener recursos para adquirir estos alimentos.

En cuanto al tipo de actividad laboral, el $69 \%$ de las mujeres era ama de casa, el $39 \%$ de los hombres desempeñaba trabajos informales, el $17 \%$ de la población estaba desempleada y consideraba que no podía trabajar debido a su condición física, solo el $3 \%$ de los desempleados consideraba que podía trabajar, y ninguno de los participantes era jubilado.

Al analizar las prácticas alimentarias, se encontró que el $96,3 \%$ observaba los tres tiempos de comida principales, solo el $59 \%$ consumía alimentos en la media mañana y el $55 \%$ en la media tarde, y el $18 \%$ consumía algún alimento después de la cena.

La mitad de los pacientes (52\%) refirió preferir las frutas enteras, el $13 \%$, frutas en jugo, y el $26 \%$, verduras; solo el $1 \%$ expresó preferencia por el consumo de snacks, de comidas rápidas, de bebidas gaseosas, de jugos industrializados o de alimentos fritos. El $92 \%$ no adicionaba sal a las preparaciones servidas y en el $94 \%$ persistía la práctica de cocinar las comidas del hogar con sal. El $78 \%$ utilizaba aceite de origen vegetal: el $49 \%$, una mezcla de aceites vegetales, el $17 \%$, aceite de girasol, el $3 \%$, aceite de oliva y el $2 \%$, aceite de canola. El $22 \%$ utilizaba grasas de origen animal, como la manteca o la mantequilla, en sus preparaciones. 
En cuanto a la frecuencia de consumo, se evidenció que el $22 \%$ no consumía avena de manera habitual y, el $34 \%$, ningún tipo de cereal integral; la frecuencia más alta de consumo de cereales integrales fue de una vez al día en $37 \%$ de los pacientes. El 57 \% consumía arroz una vez al día y, el $23 \%$, dos veces al día. En cuanto a los productos de panadería, el $41 \%$ los consumía de forma diaria, el $16 \%$, dos veces al día, y el $15 \%$, dos veces por semana. Las pastas alimenticias se consumían con menos frecuencia, pues casi el $60 \%$ de los pacientes refirió consumirlas entre una $\mathrm{y}$ dos veces a la semana.

El consumo de tubérculos y plátanos era elevado: el $45 \%$ de los pacientes los ingería por lo menos una vez al día y, el $15 \%$, entre una y cuatro veces por semana; en tanto que el de frutas y verduras era bajo: $30 \%$ de los pacientes consumía verduras una vez al día y, el $21 \%$, frutas. Solo el $2 \%$ de los pacientes consumía dos porciones de frutas 0 verduras al día.

Las carnes eran la principal fuente de proteína de alto valor biológico, aunque su consumo era bajo: solo el $50 \%$ de los pacientes consumía carne de res o pollo una vez al día y de estos, el $25 \%$ consumía carne de res con grasa visible o pollo con la piel. El $75 \%$ de los pacientes consumía pescado entre una y dos veces al mes, el $65 \%$, vísceras una vez al mes, y el $35 \%$, dos veces al mes. El consumo de huevo era habitual: el $71 \%$ lo consumía entre una y cinco veces a la semana y, el $20 \%$, diariamente.

El $38 \%$ de los pacientes consumía leguminosas (fríjol, lentejas, arvejas, garbanzos secos) entre dos y seis veces por semana, el $38 \%$, una vez por semana y, el $2 \%$, diariamente. En cuanto al consumo de lácteos, el $82 \%$ manifestó no consumir leche descremada; de los restantes, el $68 \%$ la consumía entre una y dos veces al día. El $66 \%$ tomaba leche entera; de ellos, el $57 \%$ la consumía una vez al día y, el $15 \%$, dos veces. El consumo de queso, yogur o kumis, ya fuera bajo en grasa o entero, era escaso: solo el $20 \%$ consumía estos alimentos una vez a la semana y un promedio de $75 \%$ no los consumían.

El $40 \%$ de los pacientes consumía azúcar, chocolate o panela diariamente, el $10 \%$, dos veces al día y, el $7 \%$, tres o más veces al día, en tanto que el $20 \%$ consumía postres y dulces entre una y dos veces por semana. El $91 \%$ de los participantes no consumía comidas rápidas o snacks, y solo el $20 \%$ de quienes sí lo hacían los consumían entre una y tres veces por semana. El $76 \%$ de los pacientes refirió no consumir margarina ni mantequilla, y el restante $24 \%$ las consumía entre una y cuatro veces por semana. En el cuadro 1 se presenta un resumen de la frecuencia y las prevalencias más altas de consumo.

En cuanto al manejo nutricional de estos pacientes, se encontró que el $52 \%$ de ellos refirió tener un plan dietético que incluía el aumento en el consumo de frutas (55\%) y verduras (56 \%), así como la reducción del consumo de grasas (58\%), azúcar $(80 \%)$ y sal (69 \%), pero solo el $8,9 \%$ había tenido en algún momento consulta con un profesional en nutrición.

En el cuadro 2 se observa un mayor porcentaje de personas que consumía las cinco comidas recomendadas entre quienes tenían un plan dietético (48\%) y, asimismo, la frecuencia del uso de sal fue menor (6\%) entre quienes seguían dicho plan. Se encontró una diferencia estadísticamente significativa en el consumo de azúcares entre los pacientes que tenían un plan dietético y los que no lo tenían $(p=0,026)$.

Cuadro 1. Frecuencia de consumo de alimentos en los pacientes con diabetes mellitus de tipo 2

\begin{tabular}{|c|c|c|}
\hline Alimento & $\begin{array}{l}\text { Mayor frecuencia } \\
\text { de consumo }\end{array}$ & $\begin{array}{c}\text { Porcentaje } \\
\text { de consumo }\end{array}$ \\
\hline Arroz & $45 \%$, una vez al día & 93 \\
\hline $\begin{array}{l}\text { Papa, yuca o } \\
\text { plátano }\end{array}$ & $45 \%$, una vez al día & 92 \\
\hline Huevo & $71 \%, 3-4$ veces por semana & 92 \\
\hline $\begin{array}{l}\text { Carne y pollo } \\
\text { magros }\end{array}$ & $37 \%$, una vez al día & 90 \\
\hline $\begin{array}{l}\text { Verduras y } \\
\text { hortalizas }\end{array}$ & $30 \%$, una vez al día & 89 \\
\hline Frutas & $21 \%$, una vez al día & 84 \\
\hline Avena & $20 \%$, dos veces por semana & 78 \\
\hline Leguminosas & $35 \%$, una vez por semana & 75 \\
\hline Pasta & $38 \%$, una vez por semana & 74 \\
\hline Pan & $40 \%$, una vez al día & 73 \\
\hline Cereales integrales & $37 \%$, una vez al día & 66 \\
\hline Leche descremada & $50 \%$, una vez al día & 66 \\
\hline Aguacate & $35 \%$, dos veces al mes & 66 \\
\hline Pescado & $32 \%$, dos veces al mes & 60 \\
\hline $\begin{array}{l}\text { Azúcar, chocolate, } \\
\text { panela }\end{array}$ & $40 \%$, una vez al día & 59 \\
\hline Vísceras & $34 \%$, dos veces por semana & 35 \\
\hline $\begin{array}{l}\text { Margarina, } \\
\text { mantequilla }\end{array}$ & $30 \%$, una vez por semana & 24 \\
\hline $\begin{array}{l}\text { Carne y pollo } \\
\text { grasos }\end{array}$ & $25 \%$, una vez al día & 23 \\
\hline Leche entera & $43 \%$, una vez al día & 18 \\
\hline Dulces y postres & 29 \%, una vez al día & 18 \\
\hline Snacks & $45 \%$, una vez al mes & 18 \\
\hline Comidas rápidas & $5 \%$, dos veces por semana & 9 \\
\hline
\end{tabular}


Cuadro 2. Diferencias entre conductas alimentarias

\begin{tabular}{|c|c|c|c|c|c|}
\hline \multirow[t]{2}{*}{ Conducta alimentaria } & \multicolumn{2}{|c|}{ Con plan dietético } & \multicolumn{2}{|c|}{ Sin plan dietético } & \multirow[t]{2}{*}{$\mathbf{p}$} \\
\hline & $\mathbf{n}$ & $\%$ & $\mathbf{n}$ & $\%$ & \\
\hline Cinco tiempos de comidas & 53 & 48 & 41 & 41 & 0,269 \\
\hline Consumo de aceite de girasol & 20 & 18 & 16 & 16 & 0,652 \\
\hline Consumo de aceite de oliva & 5 & 5 & 1 & 1 & 0,111 \\
\hline Consumo de manteca & 6 & 5 & 8 & 8 & 0,477 \\
\hline Consumo de mantequilla & 20 & 18 & 11 & 11 & 0,132 \\
\hline Consumo de margarina & 7 & 6 & 5 & 5 & 0,657 \\
\hline Cocinar con sal & 98 & 89 & 97 & 96 & 0,295 \\
\hline Adicionar sal a las preparaciones servidas & 7 & 6 & 10 & 10 & 0,351 \\
\hline Retirar la piel del pollo & 86 & 78 & 68 & 67 & 0,078 \\
\hline Retirar la grasa visible de la carne & 88 & 80 & 79 & 78 & 0,751 \\
\hline Consumo de frutas diario & 41 & 37 & 36 & 36 & 0,807 \\
\hline Consumo de verduras diario & 58 & 53 & 45 & 45 & 0,236 \\
\hline Consumo de azúcar, chocolate y panela & 51 & 46 & 62 & 61 & 0,026 \\
\hline
\end{tabular}

\section{Discusión}

La mayoría de los pacientes estaba en un rango de edad coincidente con el periodo de vida productivo y el de mayor riesgo de padecer diabetes mellitus de tipo $2(16,17)$. En este sentido, se evidenció que la actividad laboral se vio disminuida como consecuencia de la enfermedad, lo que afectó negativamente la calidad de vida de estas personas y su capacidad adquisitiva. En la población adulta mayor, la principal dificultad fue la dependencia con respecto a su entorno familiar o a otras personas, ya que ninguno era jubilado, lo cual dificultaba el cumplimiento de las recomendaciones nutricionales; además, la alimentación del paciente era diferente a la del resto de la familia y no se consideraba que pudiera ser adoptada por el núcleo familiar completo.

La prevalencia de la diabetes mellitus de tipo 2 en este estudio fue mayor en las mujeres que en los hombres, lo cual concuerda con estudios de otras regiones del país y de América (18-20); la información sobre por qué la diabetes afecta en mayor medida al sexo femenino es insuficiente y esta condición no se ha considerado como un factor de riesgo para la enfermedad, por lo cual es importante estudiarla con mayor profundidad. Por otra parte, según la Guía de práctica clínica para la prevención, diagnóstico y tratamiento del sobrepeso y la obesidad en adultos (21), el $90 \%$ de la prevalencia de esta enfermedad es atribuible al exceso de peso y, según los datos reportados por la ENSIN 2010 (14), en Colombia la prevalencia de exceso de peso fue de $51,1 \%$ y mayor en las mujeres $(55,1 \%)$, lo cual puede estar relacionado con una mayor prevalencia de diabetes mellitus de tipo 2 entre ellas. Puede concluirse, entonces, que si el sobrepeso y la obesidad se previenen y se tratan adecuadamente, podría reducirse de forma significativa la carga de esta enfermedad, que hoy constituye la quinta causa de mortalidad en el país (21).

Otro hallazgo relevante de esta investigación fue el bajo nivel educativo de los pacientes y el analfabetismo, lo cual afectó directa y negativamente el cumplimiento del tratamiento debido a la dificultad para leer las indicaciones y recomendaciones.

Por otro lado, se constató que la aplicación del formulario de frecuencia de consumo es una buena herramienta para estimar la frecuencia habitual de la ingestión de alimentos durante el mes anterior, lo que permite establecer el patrón aproximado de consumo de alimentos y las prácticas alimentarias en estos pacientes, así como comparar y detectar conductas de riesgo en ellos (14).

En este sentido, aun cuando la distribución del valor calórico total recomendado para estos pacientes es de 10 a $20 \%$ de proteína, 50 a $60 \%$ de carbohidratos, 30 a $35 \%$ de grasas, y 20 a $35 \mathrm{~g}$ de fibra al día (22), y se recomienda una dieta rica en ácidos grasos monoinsaturados (11), en este estudio se evidenció que en la dieta de los pacientes atendidos en estos hospitales predominaba el consumo de carbohidratos, como los almidones de los cereales y subproductos elaborados con estos, y de tubérculos y plátanos, además del consumo de azúcar y el uso de grasas perjudiciales para la salud, lo cual se asocia con el bajo costo de estos alimentos y responde al patrón cultural de los hábitos alimentarios de la población colombiana (14).

El consumo de frutas y verduras se recomienda ampliamente para el manejo de la diabetes mellitus 
de tipo 2. Hoy se recomienda el consumo de cinco porciones, o más de $400 \mathrm{~g}$ diarios, de estos alimentos a toda la población en Colombia como parte de una dieta saludable baja en grasas, azúcares y sal (o sodio) para prevenir el aumento de peso como factor de riesgo de esta enfermedad (23). Sin embargo, en los pacientes del estudio se evidenció un bajo consumo de frutas y verduras, lo que limita considerablemente el aporte de fibra, vitaminas y minerales.

En cuanto al consumo de alimentos que son fuente de proteínas de alto valor biológico, en pacientes mal controlados se ha registrado una aceleración de la gluconeogénesis al aumentarse la producción de glucosa en el estado postabsortivo o posprandial, pero la influencia en los niveles de glucemia y la sensibilidad a la insulina es mínima en pacientes con diabetes mellitus de tipos 1 y 2, independientemente del consumo de proteínas; además, no hay información suficiente para confirmar que la cantidad necesaria de proteína en pacientes con diabetes mellitus de tipo 2 sin complicaciones varíe con respecto a la recomendada en individuos sanos (22). En este sentido, en el presente estudio se registró un bajo consumo de alimentos fuente de proteínas de alto valor biológico, lo que podría influir en el deterioro progresivo del estado nutricional de los pacientes.

Por otra parte, en el presente estudio uno de los factores asociados a las inadecuadas prácticas alimentarias fue la difícil situación económica de los pacientes, ya que los bajos ingresos y el no contar con planes alimentarios adecuados a su contexto sociocultural, no les permitían acceder a una alimentación apropiada, dificultando así el control de la enfermedad. Este resultado concuerda con los de un estudio en Cuba, en el cual se encontró una asociación entre el bajo consumo de proteínas y las difíciles condiciones económicas de los pacientes, lo que se traducía en un aumento del consumo de carbohidratos por encima de las recomendaciones establecidas para esta población (24). Es claro que las dificultades económicas disminuyen el cumplimiento de las recomendaciones nutricionales como parte del tratamiento, por lo cual es prioritario que, para modificar el estilo de vida, se tengan en cuenta las características sociales, culturales y económicas de los pacientes, ya que estas influyen de forma directa en la historia natural de la diabetes mellitus de tipo 2 (25-27).

Asimismo, el estudio reveló que no todos los pacientes contaban con un plan de alimentación, ni accedían a consultas especializadas con profesionales en nutrición o a las recomendaciones básicas por parte del médico tratante en algún momento de su tratamiento. Este resultado concuerda con un estudio en Bogotá (28), en el que el $36,7 \%$ de los pacientes no había recibido información general sobre la alimentación por parte del médico tratante y el 39,4 \% no había tenido acceso a la asesoría de nutricionistas dietistas, con el obvio efecto negativo en los pacientes.

En este sentido, es importante que todos los pacientes con diabetes mellitus de tipo 2 tengan acceso a la orientación y el manejo nutricional de los profesionales en nutrición para reducir el riesgo cardiovascular, alcanzando y conservando niveles de glucemia y lipemia normales, así como para mejorar el comportamiento frente a algunas de las prácticas alimentarias prescritas para esta población, especialmente la disminución del consumo de azúcares y el fraccionamiento de la alimentación. Es esencial que los pacientes reciban el apoyo y el seguimiento adecuados de un equipo multidisciplinario que incluya la orientación psicológica en apoyo de un mejor cumplimiento del tratamiento y manejo de la enfermedad (22).

En cuanto a los programas dirigidos a estos pacientes, en estudios recientes se ha demostrado que las intervenciones de prevención de la diabetes que incluyen la dieta y el entrenamiento aeróbico y de resistencia son moderadamente eficaces para favorecer la pérdida de peso $\left(-3,79 \mathrm{~kg} ;-6,13,-1,46 ; \quad \mathrm{IC}_{95 \%} ; \mathrm{z}=3,19 ; \mathrm{p}=0,001\right)$, mejorar la glucosa plasmática en ayunas $(-0,13$ mmol.L-1[-0,24, -0,02; IC $\left.{ }_{95 \%} ; z=2,42 ; p=0,02\right)$ y la tolerancia a esta (29). Sin embargo, deben hacerse estudios con seguimiento a largo plazo para determinar la eficacia de las intervenciones con múltiples componentes en la prevención de la diabetes mellitus de tipo 2 mediante el análisis de los cambios en los biomarcadores de riesgo y la contribución específica de cada componente en la reducción de los riesgos asociados con esta enfermedad (29).

Debe resaltarse que, en este estudio, se evidenciaron barreras para el adecuado manejo nutricional y general de los pacientes, y que el bajo nivel educativo, el analfabetismo, la gran dependencia de los pacientes con respecto a sus familias, las escasas oportunidades laborales, la incapacidad percibida por los pacientes para trabajar debido a su condición y los bajos recursos económicos, dificultan un buen proceso de tratamiento y de 
prevención de las complicaciones derivadas de la diabetes. Por ello, es importante sensibilizar y motivar a los profesionales de la salud para que hagan una consulta especializada y ofrezcan un acompañamiento permanente ajustado al contexto sociocultural de los pacientes (30). Además, se ha reportado que la comunicación entre el paciente diabético y el médico o profesional tratante es un factor importante en el cumplimiento del tratamiento, y ofrece la oportunidad de construir conjuntamente con los pacientes planes de tratamiento mutuamente aceptables y de alcanzar metas acordadas, lo cual estimula al paciente a tomar decisiones beneficiosas para su salud y mejora los resultados de la intervención (31). También, se obtienen resultados positivos cuando los programas educativos incluyen refuerzos, seguimiento telefónico en casa y renegociación de objetivos con metas intermedias de control metabólico (32).

En Colombia, no hay suficiente información sobre la alimentación de los pacientes con diabetes mellitus de tipo 2 en hospitales públicos. En este sentido, el presente estudio permitió una aproximación a sus patrones de alimentación, así como determinar las prácticas alimentarias y las conductas de riesgo, por lo que sus resultados son fundamentales para las entidades e instituciones prestadoras de servicios de salud a la hora de mejorar las estrategias de prevención y control de la enfermedad y contribuir, así, a mejorar la calidad de vida y el cumplimiento del tratamiento en estos pacientes, teniendo en cuenta su contexto, la realidad en la cual están inmersos y sus propias necesidades $(33,34)$.

\section{Agradecimientos}

A la Secretaría de Salud de Cundinamarca, los Hospitales San José del municipio de Guaduas, San Rafael del municipio de Facatativá y Universitario La Samaritana de Zipaquirá.

\section{Conflicto de intereses}

Los autores del presente artículo declaramos no tener conflicto de intereses.

\section{Financiación}

Este estudio fue financiado por el Instituto Nacional de Salud.

\section{Referencias}

1. World Health Organization. Preventing chronic diseases a vital investment. Fecha de consulta: 26 de octubre de 2016. Disponible en: http://www.who.int/chp/chronic_disease_ report/contents/en/
2. American Diabetes Association. Standards of Medical Care in Diabetes. Diabetes Care. 2005;28(Suppl.1):S4-S36. https://doi.org/10.2337/diacare.28.suppl_1.S4

3. Instituto Nacional de Salud, Observatorio Nacional de Salud. Tercer Informe ONS: mortalidad evitable en Colombia para 1998-2011. Bogotá, D.C.: Imprenta Nacional de Colombia; 2014. Fecha de consulta: 11 de octubre de 2016. Disponible en: https://www.minsalud.gov.co/sites/ rid/Lists/BibliotecaDigital/RIDE/IA/INS/Informe3-ME-ONSDefinitivo.pdf

4. American Diabetes Association. Economic costs of diabetes in the U.S. in 2012. Diabetes Care. 2013;36:103346. https://doi.org/10.2337/dc12-2625

5. Secretaría Distrital de Salud de Bogotá, Asociación Colombiana de Diabetes. Norma guía para el programa de prevención y control de la diabetes mellitus para Bogotá, D.C. Primera edición. Bogotá: Litográficas Velasco; 2004. p. 7.

6. Lockwood D, Frey ML, Glandish NA, Hiss RG. The biggest problem in diabetes. Diabetes Educ.1986;12:30-3. https://doi.org/10.1177/014572178601200107

7. Román DA, Bellido D, García P, Gamboa R, Alcázar V, De la Fuente $\mathbf{R}$, et al. Dietoterapia, nutrición clínica y metabolismo. Segunda edición. Madrid: Ediciones Díaz de Santos; 2012. p. 696-9.

8. Liu S, Manson JE, Stampfer MJ, Hu FB, Giovannucci E, Colditz GA, et al. A prospective study of whole-grain intake and risk of type 2 diabetes mellitus in US women. Am J Public Health. 2000;90:1409-15.

9. Vessby B. Dietary fat and insulin action in humans. $\mathrm{Br}$ J Nutr. 2000;83(Suppl.1):S91-6. https://doi.org/10.1017/ S000711450000101X

10. Hu FB, van Dam RM, Liu S. Diet and risk of type II diabetes: The role of types of fat and carbohydrate. Diabetologia. 2001;44:80517. https://doi.org/10.1007/s001250100547

11. Aguilera CM, Ramírez-Tortosa CM, Mesa MD, Gil A. Efectos protectores de los ácidos grasos monoinsaturados y poliinsaturados sobre el desarrollo de la enfermedad cardiovascular. Nutr Hosp. 2001;16:78-91.

12. Santiago J. Lessons from the diabetes control and complications trial. Diabetes. 1993;42:1549-50. https://doi. org/10.2337/diab.42.11.1549

13. Ministerio de la Protección Social. Guía de atención de la diabetes mellitus tipo 2. 2005. Fecha de consulta: 26 de octubre de 2016. Disponible en: http://www.medinformatica.net/TERAPEUTICA-STAR/DiabetesTipo2_ GuiaAntencionMPS_guias17.pdf

14. Profamilia, Instituto Nacional de Salud, Instituto Colombiano de Bienestar Familiar, Ministerio de Salud y Protección Social. Encuesta Nacional de la Situación Nutricional en Colombia, ENSIN. Bogotá: Da Vinci editores \& Cia.SNC; 2010

15. Instituto Colombiano de Bienestar Familiar. Guías alimentarias basadas en alimentos para la población mayor de 2 años. Primera edición. Bogotá: ICBF; 2014.

16. International Diabetes Federation. Atlas de la diabetes de la FDI. Fecha de consulta: 10 de octubre de 2016. Disponible en: http://www.fundaciondiabetes.org/upload/ publicaciones_ficheros/95/IDF_Atlas_2015_SP_WEB_ oct2016.pdf 
17. Barceló A, Rajpathak S. Incidence and prevalence of diabetes mellitus in the Americas. Pan Am J Public Health. 2001;10:300-8. https://doi.org/10.1590/S102049892001001100002

18. Aschner P, King H, Triana M, Rodríguez BM. Glucose intolerance in Colombia. A population-based survey in an urban community. Diabetes Care. 1993;16:90-3.

19. Escobedo J, Buitrón L, Velasco M, Ramírez J, Hernández $\mathbf{R}$, Macchia A, et al. High prevalence of diabetes and impaired fasting glucose in urban Latin America: The CARMELA Study. Diabet Med. 2009;26:864-71. https://doi. org/10.1111/j.1464-5491.2009.02795.x

20. Cabrera-Pivaral CE, Martínez-Ramírez A, Vega-López MG, González-Pérez G, Muñoz-de la Torre A. Prácticas nutricias en pacientes diabéticos tipo II en el primer nivel de atención. Instituto Mexicano del Seguro Social (IMSS), Jalisco, México. Cad Saúde Públ. 1996;12:525-30. https:// doi.org/10.1590/S0102-311X1996000400011

21. Ministerio de Salud y Protección Social, Colciencias. Guía de práctica clínica (GPC) para la prevención, diagnóstico y tratamiento del sobrepeso y la obesidad en adultos. 2016. Fecha de consulta: 16 de octubre de 2016. Disponible en: http://gpc.minsalud.gov.co/gpc_sites/Repositorio/Conv_637/ GPC_obesidad/GUIA_SOBREPESO_OBESIDAD_ADULTOS_ COMPLETA.pdf

22. Cánovas B, Koning AM, Muñoz C, Vázquez C. Nutrición equilibrada en el paciente diabético. Nutr Hosp. 2001;16: 31-40.

23. Ministerio de Salud y Protección Social. Documento Guía Alimentación Saludable. Fecha de consulta: 26 de octubre de 2016. Disponible en: https://www.minsalud.gov. co/sites/rid/Lists/BibliotecaDigital/RIDE/VS/PP/SNA/GuiaAlimentacion-saludable.pdf

24. Blanco J, Socarrás MM, González D, Licea M. Algunos indicadores de la dieta en un grupo de pacientes diabéticos tipo 2 de Centro Habana. Revista Cubana Aliment Nutr. 2002;16:23-30.

25. López-Carmona JM, Araiza-Andraca CR, RodríguezMoctezuma JR, Munguía-Miranda C. Construcción y validación inicial de un instrumento para medir el estilo de vida en pacientes con diabetes mellitus tipo 2. Salud Pública Mex. 2003;45:259-68. https://doi.org/10.1590/S003636342003000400004

26. Maya LH. Los estilos de vida saludables: componente de la calidad de vida. Fecha de consulta: 15 de diciembre de 2016. Disponible en: https://www.funlibre.org/documentos/ lemaya1.htm

27. Wubben DP, Porterfield D. Health-related quality of life among North Carolina adults with diabetes mellitus. N C Med J. 2005;66:179-85.

28. Barrera MP, Pinilla AE, Caicedo LM, Castillo YM, Lozano YM, Rodríguez KM. Factores de riesgo alimentarios y nutricionales en adultos con diabetes mellitus. Rev Fac Med. 2012;60:S28-40.

29. Aguilar EJ, Morgan PJ, Collins CE, Plotnikoff RC, Callister R. Efficacy of interventions that include diet, aerobic and resistance training components for type 2 diabetes prevention: A systematic review with metaanalysis. Int J Behav Nutr Phys Act. 2014;11:2. https://doi. org/10.1186/1479-5868-11-2

30. Ockleford E, Shaw RL, Willars J, Dixon-Woods M. Education and self-management for people newly diagnosed with type 2 diabetes, a qualitative study of patients' views. Chronic Illn. 2008;4:28-37. https://doi.org/10.1177/1742395307086673

31. Matthews SM, Peden AR, Rowles GD. Patient-provider communication: Understanding diabetes management among adult females. Patient Educ Couns. 2009;76:31-7. https://doi.org/10.1016/j.pec.2008.11.022

32. Maldonato A, Bloise D, Ceci M, Fraticelli E, Fallucca F. Diabetes mellitus: Lessons from patient education. Patient Educ Couns. 1995;26:57-66. https://doi.org/10.1016/07383991(95)00736-J

33. Trinidad I, Fernández J, Cucó G, Biarnés E, Arija V. Validación de un cuestionario de frecuencia de consumo alimentario corto: reproducibilidad y validez. Nutr Hosp. 2008;23:224-52.

34. López-Amador K, Ocampo-Barrio P. Creencias sobre su enfermedad, hábitos de alimentación, actividad física y tratamiento en un grupo de diabéticos mexicanos. Arch Med Fam. 2007;9:80-6. 